\title{
An intelligent robots-server system for solar panel cleaning and electric power output optimization
}

\begin{abstract}
Electric energy production from solar panels is becoming more and more economically viable as the efficiency of the new solar panels increases and their cost decreases steadily. Solar panels in places where the sun shines almost every day of the year provide a clean alternative to traditional electric energy generators, without placing stress on the environment. Large scale solar panel installations could lose a percentage of their output due to dust, bird droppings, cable destruction by small animals with sharp teeth, panel destruction by vandals, and many other reasons. In this research paper we provide an improvement of our robots server architecture. This improvement is in the intelligence of the robots, the server, and the installation of four propellers on the mechanical structure of the robot to enable it to jump, or perform short flights. The project is still in the experimental stage and it will take some changes and some time until it becomes fully functional and economically feasible to become a consumer product.
\end{abstract}

Keywords: convolutional neural networks, computer vision, secure communications
Volume 3 Issue 5 - 2017

\section{Evangelos Yfantis}

Computer Science Department, University of Nevada, USA

Correspondence: Evangelos Yfantis, Computer Science Department, University of Nevada, USA, Tel 89154-4019, Email yfantis@cs.unlv.edu

Received: July 13, 2017 | Published: November 20, 2017

\section{Introduction}

As solar panels are becoming economically viable more large scale solar power installations are sprouting throughout the world, producing gigawatts per hour energy that is relatively inexpensive and pollution free. In arid environments where the sun shines every day, except on rare occasions of rain, solar power installations in arid environments when the sun is shining every day except in the rare occasions solar power installations are very productive. Since the inter arrival time between rainfalls is relatively long, strong winds stir up dust that settles on the surface of the solar panels and accumulates over time, inhibiting the solar ray penetration needed to produce the maximum electric energy possible for each panel. In addition migrating birds stop at the solar energy facilities to rest and may deposit droppings which inhibit energy production and chemically react with the surface of the solar panel if they remain on the panel surface for a long time. This alters the surface so that the new surface is not as efficient in absorbing the sunlight, and thus the solar panel produces less energy. Since large scale solar panel installations are placed in large empty areas outside a city, they are subject to the destruction by wild animals that inhabit these areas. Thus wild animals with sharp teeth often times cut the cables of the solar panels. Solar panels provide shading from the sun during the very hot summer days. Animals take advantage of this protection and often times create nests underneath the panels. These animal nests along with the animal activities accelerate the wear and tear of the solar panel cables. Solar panel shading also protects vegetation which often times grows tall enough to interfere with the solar panels and reduce their output, or even change the panel orientation due to the interference of the roots with the base of the panel installation. In addition to that human vandalism could result in breaking of the panel glass or creating a small gap between the air tight glass and the frame, allowing moisture, fungus, ants, and other things to settle in and inhibit the solar panel output. From the above we infer that solar panels need maintenance. The maintenance includes inspection to ensure that the cables are not torn and in very good condition, detection and removal of vegetation so that it will not cause future problems, the panels and cleaning of the panels so they perform at the maximum of their ability. The more employees a solar panel facility has the higher the cost of a $\mathrm{kWh}$ becomes. Inconsistencies during cleaning create stripes on the surface panels that inhibit power production until corrected in the next scheduled cleaning. Placing heavy objects during cleaning on the panel glass, could partially disconnect the glass from the frame, creating a small gap that enables the moisture to settle in, and fungus, ants, other small creatures that inhibit the energy output of the panels. Other mistakes during cleaning could result into cracking or breaking the solar panel glass. Automation has the ability to provide better quality maintenance, that is less expensive, more consistent, and optimize the electric energy output. The system we have been working on is a network of small robots, having secure wireless connection with a server. The communication between server-robots is real time via network sockets. Depending on the size of the solar panel installation and the antenna network placed in the installation, the wireless protocol used is either $\mathrm{Wi}-\mathrm{Fi}$, or the industrial-scientific-medical (ISM). This research paper is structured as follows: Abstract, followed by Introduction, followed by the system description, followed by the hardware description, followed by the software description, followed by conclusions, and finally the references.

\section{The system}

The system consists of a Linux raid array file server with hot swap, and a set of robots. The server consists of our software which includes a multithreaded communication program that enables the server to get signals from each robot. The signals transmitted by the robots to the server include the GPS position of each robot, real time video obtained by the robot, decision if a solar panel is dirty or clean and if a panel has been cleaned, or is left as is until the next inspection. Also, if the cables are in good condition or in need of repairs by the designated repair people. This information is also followed by pictures of the cables. The signals the server sends to the robot are: to turn the motors on or off, to change the speed of one or more motors, to move in a certain direction, to change direction (turn), to engage the propellers in order to fly, to stand still at a certain altitude, to move in a certain 
direction while in flight, to land in a certain location which is on the camera's line of sight. The robot has an intelligent system on board which takes the inputs of the image of the solar panel obtained by the four cameras which are part of the vision system of the robot, performs digital image processing ${ }^{1}$ and decides if the panel is dirty enough to need cleaning, ${ }^{2}$ or if it is not dirty enough and the cleaning should be postponed for the next inspection. If the robot decides the panel needs cleaning a small rotating brush working together with a small vacuum is activated that cleans the solar panel in a progressive way similar to the way that the old dot matrix printers used to be operating. The information pertaining to the status of the solar panel are transmitted back to the file server and are used by the file server to update the status of the particular solar panel in the data base. Once a solar panel is finished the file server gives the robot the next set of instructions. The file server also keeps track of the amount of time remaining before the battery is empty enough that the robot cannot operate, and if needed instructs the robot to return to the robot station in order to plug in to an available solar panel used to fill the battery. Robots in the station waiting for their battery to get filled are logged in the data base as unavailable. Once a robot's battery is full then the status of that robot in the data base changes from unavailable to available, providing that the robot is functional. If the robot is not functional a repairman is scheduled to repair the robot and the robot becomes unavailable until fully repaired and fully charged in which case the robot becomes available again. The file server therefore consists of three subsystems. The secure communication system ${ }^{3,4}$ between server and each robot. The navigation instruction system that guides each robot. The data base system that keeps track real time the status of each robot, the location, the function that the robot performs currently, and the status of each panel. Each robot has the following software: communication between the robot and the server, embedded software in microcontrollers driving the electronic speed controllers, taking the input from the GPS, performing the message packetization, and transmission. Additional software performing video compression, digital image processing, and artificial intelligence ${ }^{5}$ for detecting if a panel needs cleaning or not, resides in FPGAs. Finally additional software embedded in microcontrollers controlling the vacuum system of the robot and the brush is part of the robot system. The communication between server and the robots is encrypted using the encryption of the TLS system in Linux. The hardware part of the system consists of Linux Raid array 5 file servers with hot swap and 12 terra bytes of disk space, a large percentage of which is devoted to video. The video is compressed with H.265 and is stored in a circular fashion which means that after certain time period the new video replaces the oldest video. The video software enables the user to look at the video forward or backward at any specified time for any camera, for any period of time. The hardware part also includes the robots, the router, and the antenna system used to relay the wireless signals to the server from a robot, or to a robot from the server. The robot consists of the tank-like wheel system powered by two electronic motors, each one of them connected to its own electronic speed controller, a vacuum system that includes the vacuum and the brush, four cameras that are part of the robot vision system, four propellers with four motors and four electronic speed controllers, a PCB board to control the motion of the robot, control the vacuum and brush, perform video compression, digital image processing, $\mathrm{AI}$ and transmission. In the following sections the software and hardware are described separately in more detail.

\section{The hardware}

The hardware consists of: a Linux file server configured into a Raid 5 array with hot swap, which means in case of a disk failure the disk can be replaced while the server is running, and the redundancy of the Raid 5 is capable of restoring the new disk configuration into its original form. It also consists of the ever evolving robot named Helios (the Greek name for Sun). The first generation of the robot Helios-1, is depicted in Figure 1. The tank-like design of the motion system of the robot is so that is able to move in rough terrain. In the top of the tank there is a telescopic system with four cameras. The imagers of the cameras are high quality CMOS. The body of the robot includes two motors to move the left and right wheel. Each one of the two motors is connected to an electronic speed controller that takes signals from a microcontroller and by controlling the speed of the right motor and left motor enables the robot to turn left or right or move forward. The microcontroller takes the instructions either from the file server or from the vision-motion system of the robot. If the vision system detects rough terrain that needs to be negotiated the intelligent.

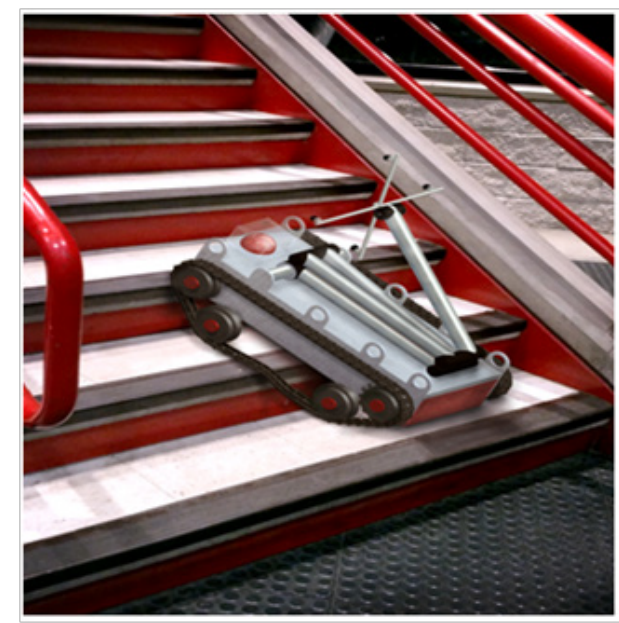

Figure I Helios-I Robot, designed for rough terrain.

System instructs the microcontroller how to control the electronic speed controllers so that the robot can overcome the obstacle. The body of the robot includes also a motor that drives the telescopic vision system, and places it either above the solar panel to inspect the panel, or below the solar panel to inspect the cable connections, as well as for growing vegetation, animal activity (animal nesting, animal droppings, animal presence). The body of the robot also includes a PCB board with FPGAS (so that can be reprogrammed) holding the image processing of the images obtained by the cameras during inspection, and the intelligent system that classifies the surface of a solar panel as clean or need to be cleaned. The same vision-intelligent system detects if cables are damaged and need to be replaced or repaired, or not damaged (Figure 2). It also detects animal activity, and vegetation growth. The lower center part of the robot includes another motor driving the vacuum, and another motor next to it driving the rotating brash. The design of the next generation Helios-II, includes four folding propellers each one driven by its own motor that enable the robot to perform very short flights when needed to jump in the top of a panel that is high above the ground. 


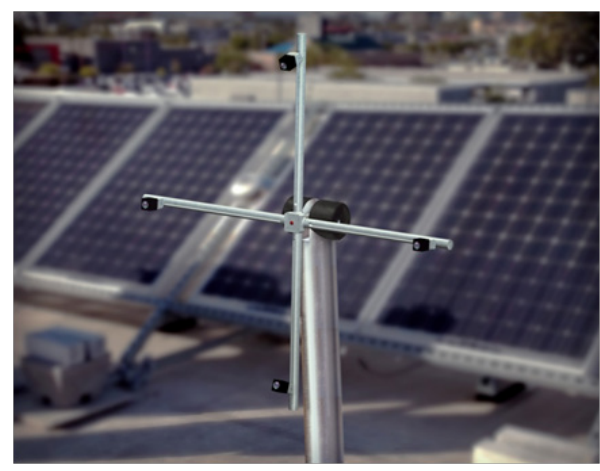

Figure 2 Helios-I, Robot-Vision System.

\section{The software}

The file server software consists of several subsystems: The communications software is written in visual $\mathrm{C}++$ using network sockets. It includes the video management software. For each incoming video a new thread is created and a new port is opened. For each robot two additional ports need to be open, one for server to robot instructions and one for robot to server messages. It also includes the data base management software. The data base software is using MySQL, with PHP. The data base is based on the entity relationship model and includes several tables: A table about the robots, which includes the robot id, robot location, robot activity, and battery charge percent. A panel table, which includes when last inspected, cleaned or not, cable status from last inspection, glass status from last inspection, energy output, and next scheduled inspection. Repair-people data base which includes their success rate in various kinds of robot repairs, and solar panel repairs. Rule based artificial intelligence software, is also included in the server. This software enables the file server to get the GPS coordinate of a robot correlate it with the video from the robot's cameras, and understand exactly where the robot is. It uses this information to guide the robot real time thru each path until it reaches each first inspection destination. The robot software includes communication with the server, which includes video transmission to the server, information related to the panel it currently inspects, and information related to the panel it just cleaned. The robot also has local intelligence. The robot intelligent system gets the digitally processed camera images as input to a convolutional neural network and decides if the solar panel does or does not need to be cleaned. If does not need to be cleaned it sends that information to the server, the server records the information to the data base, checks the percent charge of the battery and if there is not enough charge it instructs the robot to proceed to the charge station otherwise it instructs the robot to inspect the next panel. If the currently inspected panel needs cleaning the robot by the aid of its vision system and digital image processing finds the left top corner of the panel, activates the rotating brash and the vacuum system, and proceeds to clean the panel in a raster way from the top until it reaches the right bottom Once the panel is cleaned it sends a message to the server that the panel was cleaned. The server updated the panel record specifying the date and time it was cleaned, and send instructions to the robot what to do next. The robot software also includes embedded software to microcontrollers. When the robot moves the majority of the time does not fly, but moves in the ground like a small tank with the aiid of two motors. Each one of these two motors is controlled with the aid of an electronic speed controller which is programmed to change the speed of the motor connected to. Thus of the speed of the right motor is less than the speed of the left motor the robot turns right. If the speed of both motors is the same the robot moves forward.

\section{Conclusion}

A file server robot Architecture was described in this research. The general concept of the architecture is viable and if produced at low enough cost could keep the solar panels clean increasing their life expectancy, and increasing their energy output. The architecture is still in an experimental stage and the details of the hardware and software are still evolving. Proof of concept system that we have developed so far have shown us that this architecture is capable of providing the automation needed to maintain the solar panels the way we described it in this research paper.

\section{Acknowledgments}

No acknowledgement.

\section{Conflict of interest}

No conflict of interest.

\section{References}

1. Canny JF. A Computational approach to edge detection. IEEE Transactions on Pattern Recognition and Machine Intelligence. 1986;8(6):679-698.

2. Zamora Ramos E, Ho S, Yfantis EA. Using spectral decomposition to detect dirty solar panels and minimize impact on energy production. Journal of Advances in Image and Video Processing. 2015.

3. Diffie W, Hellman M. New directions in cryptography. IEEE Trans Information Theory. 1976;22(6):644-654.

4. Y fantis EA, Nakakuni M, Zamora Ramos E. Low-bandwidth transmission algorithm for reliable wireless communication. The $7^{\text {th }}$ IEEE Annual CCWC. 2017. p. 38-42.

5. Zamora Ramos E, Nakakuni M, Yfantis EA. Quantitative Measures to Evaluate Neural Network Weight Initialization Strategies. The $7^{\text {th }}$ IEEE Annual CCWC. 2017. p. 31-37. 